\title{
Annotating the discourse and dialogue structure of SMS message conversations
}

\author{
Nianwen Xue, Qishen Su, Sooyoung Jeong \\ Brandeis University \\ Computer Science Department \\ $\{$ xuen, qsu, jeong\}@brandeis.edu
}

\begin{abstract}
In this paper we present a framework for annotating the discourse and dialogue structure of SMS message conversations. The annotation specifications integrate elements of coherence-based discourse relations and communicative acts in conversational speech. We present annotation experiments that show reliable annotation can be achieved with this annotation framework.
\end{abstract}

\section{Introduction}

With the pervasive use of mobile devices, Short Message Service (SMS) has been used widely in day-to-day communications. In many cases SMS messages have taken the place of traditional telephone conversations, and have become the preferred method for people to communicate with one another. SMS messages are by definition short, and due to its asynchronous nature, a participant does not have to wait to respond before another participant finishes. As a result, it is often the case that the conversation does not alternate in a rigid manner between participants.

The relations between the messages in an SMS conversation are in some ways very similar to those between utterances in conversational speech, where a conversant may agree or disagree with, respond to, or indicate understanding (or nonunderstanding) of an utterance by another speaker. To the extent that they are similar, the relations between SMS messages can be characterized in terms of the dialogue annotation framework described in (Core and Allen, 1997). The dialogue structure of the SMS "conversations" also tends to be more complex than that of speech conversations as a result of the more complex turn-taking patterns in SMS messages.
SMS message conversations are also different from conversational speech in that they are primarily in text form. Text within a single message also demonstrates the kind of discourse coherence that is typical of written text.

In this paper we describe a framework for annotating the discourse and dialogue structure of SMS message conversations. Based on the linguistic characteristics of SMS messages, we design an annotation framework that integrates elements of dialogue and discourse annotations, and report experiments that show reliable annotation with this framework.

The rest of the paper is organized as follows. In Section 2, we describe our annotation framework in detail. In Section 3 we report results on annotation experiments that show reliable annotation, and we will also discuss sources of disagreement. In Section 4 we discuss related work. We conclude our paper and describe future work in Section 5.

\section{Annotation framework}

In this section, we describe key elements of our annotation framework. We first describe basic units of our annotation, and then discuss how the basic units relate to each other to form a dialogue structure. Finally we present the set of relations we use in interpreting this structure.

\subsection{Units of annotation}

The basic units of annotation are individual text messages. The SMS messages are usually short, and most of the messages consist of single sentences, but there are a small and yet significant proportion of messages that consist of multiple sentences. In our current round of annotation, we do not analyze relations between the sentences inside one message, but we leave that possibility open for future rounds of annotation. Compared 
with discourse annotation of newswire text (Carlson et al., 2001; Prasad et al., 2008), determining the text units to perform annotation on is a relatively simple task, due to the fact that there is a natural boundary between text messages.

\subsection{Structure of the SMS message conversations}

Due to the asynchronous nature of SMS message conversations, individual messages are often "out of order", and determining which message relates to which is a substantial part of the annotation. This aspect of the annotation is different from the annotation of newswire texts or even conversational speech, where the "normal" order is generally maintained, although in conversation speech, there are often interruptions that break the normal pattern of turn-taking (Stolcke et al., 2000). Although there are some exceptions, in general, we assume that one message is only related to one previous message. ${ }^{1}$ We call the message we are annotating the "anaphor", and the previous message that it relates to the "antecedent". Because the messages are "scrambled", the antecedent of a message is not always the immediately previous one, although it is in most cases. In addition, the antecedent of a message may not always be from a different participant. A participant may respond to a prior message by another participant, or continue his/her own line of thought without responding to an outstanding message from the other participant. A short snippet of an SMS message conversation is presented in Figure 1. On the left side of the figure is a graph that shows how the messages are connected. Each message is identified by a numerical number followed by a letter indicating the ID of the participant. For example, "7b" indicates message No 7 by participant "b". As should be clear from the graph, some messages (e.g., 7b,12b,14a, 15b,16a, 17b) are not linked to an immediately previous message, and some messages are connected to a previous message by the same participant. The graph shares many properties of a dependency tree in that there is a single root, and each anaphor is connected to one antecedent. It also more constrained than a dependency tree at the syntactic level in that the antecedent is always before the anaphor. The dependency tree is non-projective, since if all the

\footnotetext{
${ }^{1}$ The assumption always holds except for a negligible number of cases where one message responds to multiple previous messages.
}

arcs are drawn one one side, there will be crossing edges. These properties are important in fashioning a strategy for parsing this structure automatically, a topic that is out of the scope of this paper. Linking each message to its antecedent message is the first step of our annotation project.

\subsection{Relations between the messages}

The second aspect of our annotation is to label the edges in graph, that is, to determine the relationship between each pair of connected messages. When annotating these relations, we make the distinction between same-participant message pairs and different-participant message pairs. The relations we use to label same-participant message pairs are drawn from the discourse relations defined in the Penn Discourse TreeBank (Prasad et al., 2008), but some PDTB relations are nonexistent in the SMS data. For example, we did not find cases of temporal relations in our SMS conversation data. This makes senses, since there is not much narrative text in SMS messages as there is in newswire such as Wall Street Journal articles in the PDTB and as a result, temporal relations are mostly unnecessary. On the other hand, there are also relations not covered in the PDTB. For example, there are cases where a participant uses another message to complete a previous message, presumably because s/he hit the "send" button in the middle of a message and later had to complete that message. There are also messages used to correct spelling mistakes of a previous message from the same participant. Such cases are not attested in carefully edited newswire text but they need to be accounted for in our annotation. The complete list of same-participant relations are presented in Table 3 .

The different-participant relations are drawn from DAMSL (Core and Allen, 1997), a coding scheme for annotating communication acts in conversational speech. DAMSL is a multilayer annotation framework that annotates both forward and backward communicative functions. Since we focus on the relation between the current message and its antecedent, we limit ourselves to mostly annotating backward communicative functions. The set of different-participant relations are provided in Table 2. Two of our labels, directives:request and directives:suggestion may bear some resemblance to the forward communicative functions in DAMSL, but they are used to label 


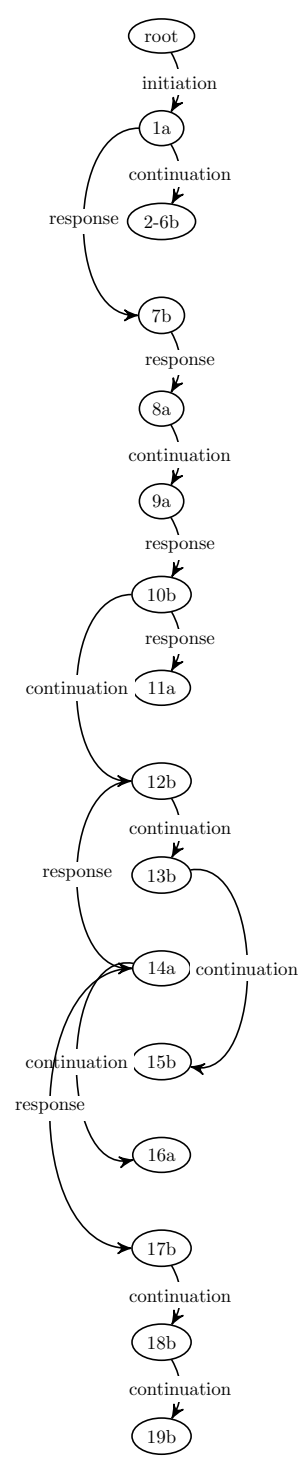

When does the ring come into the picture? you holding it off for a while

if we were going to stay in DC then I would say it is a lot closer

why would it be harder if you guys are thinking about going to NYC??

you guys would be doing this all together?

but i have no idea if $\mathrm{i}$ am going to be able to handle NYC

true

and she might not want to come back to DC if i hate it there

i know that doesn't make a lot of sense

you think she would choose the city over you? I doubt that

but pretty much no matter what happens there are going to be some sacrificest

I mean totally cool either way

no i dont think she would choose anything over me

and i wouldnt do either

which is why the situation is blurry

Figure 1: The SMS message as a dependency tree. The suffixes "a" and "b" on the tree nodes are the two participants. Messages in boxes have non-local (not immediately before) antecedents.

requests or suggestions in the context of a previous message. The following example is a case of directive:suggestion:

(1) A: I'm hungry.

B: let's go get some food!

It is important to note that unlike DAMSL, the targets of our annotation project are not individual utterances but are relations between pairs of messages. When labeling the backward communicative functions of an utterance in DAMSL, the antecedent of the utterance is assumed to be the immediately previous one, but we cannot make this assumption in our annotation.

There is a third group of labels that don't fit nicely into either group of same-participant or different-participant labels. Those labels are used to label messages that initiate a new topic, get attention, or fulfill a social obligation. These messages are explained in Table 4.

\section{Annotation Experiments}

The SMS data we performed our annotation experiments on are drawn from an LDC collection of SMS and Chat Messages collected under the DARPA BOLT program. Two annotators performed four rounds of annotation, working on the same documents so that inter-annotator agreement (IAA) statistics can be computed. We started with an initial set of guidelines. After each round of annotation, the annotators met and discussed cases of disagreement. If the differences are due to un- 


\begin{tabular}{|c|c|}
\hline Label & Description \\
\hline Agreement:Acceptance & $\begin{array}{l}\text { Acceptance refers to a positive response to proposals, requests, } \\
\text { and suggestions, or agreement to assertions. Common key words } \\
\text { of acceptance are "yes", "ok", "alright", etc. }\end{array}$ \\
\hline Agreement:Rejection & $\begin{array}{l}\text { Rejection indicates a negative response to proposals, requests, } \\
\text { and suggestions, or disagreement to assertions. Rejection is of- } \\
\text { ten signaled by words like "no" or "nah". }\end{array}$ \\
\hline Understanding:Acknowledgment & $\begin{array}{l}\text { Acknowledgment signals a participant's understanding of a pre- } \\
\text { vious message. Cue words or phrases for Acknowledgment in- } \\
\text { clude "ok", "I understand", "yes", "I know", "I see", etc. Ac- } \\
\text { knowledgment may also contain words or short phrases that ex- } \\
\text { press sentiment such as happiness, excitement, sadness, anger. } \\
\text { These words or phrases can be laughing words (such as "haha" } \\
\text { and "lol"), words that express surprise or excitement (such as } \\
\text { "omg" or "yay") and appreciation (such as "awww"), profanity } \\
\text { (such as "what the hell"), or emoticons. }\end{array}$ \\
\hline $\begin{array}{l}\text { Understanding:Non- } \\
\text { Understanding }\end{array}$ & $\begin{array}{l}\text { Non-understanding is used when a participant seeks clarification } \\
\text { by asking clarification questions. }\end{array}$ \\
\hline Directive:Request & $\begin{array}{l}\text { This relation is used when a participant asks another participant } \\
\text { to perform certain action. The immediate information or context } \\
\text { of Request, as opposed to Derivative Request, comes from the } \\
\text { other participant's message. }\end{array}$ \\
\hline Directive:Suggestion & $\begin{array}{l}\text { This relations is used when a participant offers another partici- } \\
\text { pant an idea or plan for consideration. The immediate informa- } \\
\text { tion or context of Suggestion, as opposed to Derivative Sugges- } \\
\text { tion, comes from the other participant's message. }\end{array}$ \\
\hline Question & $\begin{array}{l}\text { This relation is used to mark requests of information and clarifi- } \\
\text { cation. Unlike the clarification questions mentioned previously, } \\
\text { this type of question does not signal non-understanding. Instead } \\
\text { it is a general request for additional information. The immedi- } \\
\text { ate information or context of Question, as opposed to Derivative } \\
\text { Question, comes from the other participant's message. }\end{array}$ \\
\hline Answer:Answer & $\begin{array}{l}\text { An answer provides complete or partial information to a ques- } \\
\text { tion in a previous text message. }\end{array}$ \\
\hline Answer:Hold & $\begin{array}{l}\text { A participant sometimes signals their acknowledgment of a } \\
\text { question, but does not provide an answer to it. Moreover, if } \\
\text { a participant responds to another participant's question with a } \\
\text { question, such a response is considered as Hold }\end{array}$ \\
\hline Feedback & $\begin{array}{l}\text { This type of relation is used when a participant provides infor- } \\
\text { mation in response to another participant's message that is nei- } \\
\text { ther a question nor a directive. }\end{array}$ \\
\hline
\end{tabular}

Figure 2: Dialogue Only Labels

clear instructions in the guidelines or unclear distinctions in the tagset, the guidelines are revised before the next round of annotation starts. We made sure that the document sizes and the number of messages that we annotate in each round stay constant so that we can observe the trend in the agreement statistics after each round of anno- tation. Before we discuss the IAA, we first present the distribution of the distances between each message and its antecedent in Table 5. The distance is computed by pooling the two sets of annotations by the two annotators. The results show that overall there is a distance of 1 for only $77.97 \%$ of the message pairs, meaning that the antecedent mes- 


\begin{tabular}{|c|c|}
\hline Contingency:Cause & $\begin{array}{l}\text { Description } \\
\text { Cause indicates that the situations in two text messages influ- } \\
\text { ence each other causally, and they are not in a conditional rela- } \\
\text { tion.(Group, 2008) This type of relation is used when the argu- } \\
\text { ment of the previous message is the result, and that of the follow- } \\
\text { ing message is the cause. }\end{array}$ \\
\hline Contingency:Result & $\begin{array}{l}\text { Similar to the Cause relation, Result also indicates that the two } \\
\text { arguments have a causal relation, and that they are not in a con- } \\
\text { ditional relation. Result is used when the argument of a given } \\
\text { message is the result caused by the situation of a previous mes- } \\
\text { sage. }\end{array}$ \\
\hline Contingency:Condition & $\begin{array}{l}\text { Two text messages are in a conditional relation when the argu- } \\
\text { ment of one message is the condition and that of the other mes- } \\
\text { sage is the consequence. }\end{array}$ \\
\hline Expansion:Elaboration & $\begin{array}{l}\text { A text message is considered as an elaboration of a previous one, } \\
\text { when the current message clarifies or elaborates on the informa- } \\
\text { tion that the previous message conveys. This relation can apply to } \\
\text { two or more messages that are connected by conjunctions "and" } \\
\text { and "but". }\end{array}$ \\
\hline Expansion:Derivative Question & $\begin{array}{l}\text { This type of relation concerns with requests of information and } \\
\text { clarification, similar to Question. However, the immediate infor- } \\
\text { mation or context of Derivative Question, as opposed to Question, } \\
\text { derives from the same participant's own messages. }\end{array}$ \\
\hline Expansion:Derivative Suggestion & $\begin{array}{l}\text { This type of relation is used when a participant provides another } \\
\text { participant an idea or plan for consideration of a future action, } \\
\text { and its information or context derives immediately from the same } \\
\text { participant's own messages. }\end{array}$ \\
\hline Expansion:Derivative Request & $\begin{array}{l}\text { This elation is used when a participant asks another participant to } \\
\text { perform certain action, but its immediate information or context } \\
\text { derives from the same participant's messages. }\end{array}$ \\
\hline Expansion:Concession & $\begin{array}{l}\text { This type of discourse relation is used to highlight prominent } \\
\text { differences between two text messages. More specifically, "the } \\
\text { highlighted differences are related to expectations raised by one } \\
\text { argument which are then denied by the other"(Group, 2008). }\end{array}$ \\
\hline Expansion:Alternative & $\begin{array}{l}\text { This discourse relation is used when two text messages describe } \\
\text { alternative situations. "or", "instead" and "otherwise" are com- } \\
\text { mon cue words for this relation. }\end{array}$ \\
\hline Expansion:Completion & $\begin{array}{l}\text { Occasionally when a participant uses two or more messages to } \\
\text { complete a sentence, and Completion is used to describe the rela- } \\
\text { tion between these messages. }\end{array}$ \\
\hline Reflexive Feedback & $\begin{array}{l}\text { This relation is used when a participant answers their own ques- } \\
\text { tions or responds to their own statements (such as laughing at } \\
\text { their own joke). }\end{array}$ \\
\hline Correction & $\begin{array}{l}\text { Correction is generally concerned with correcting wrong infor- } \\
\text { mation from a previous text message, such as typos. }\end{array}$ \\
\hline
\end{tabular}

Figure 3: Non-Dialogue Only Labels

sage is the immediately previous message in only $77.97 \%$ of the cases. For the remaining $22.03 \%$ of the cases, the antecedent is not the immedi- ately previous message, indicating there is a significant proportion of messages that do not follow the "normal" order of turn-taking. The amount 


\begin{tabular}{l|l} 
Label & Description \\
\hline Topic Introduction & $\begin{array}{l}\text { It is used when a participant initiates a new topic in a new or existing } \\
\text { conversation. }\end{array}$ \\
\hline Attention Getter & $\begin{array}{l}\text { An Attention Getter is a word or phrase used to attract the attention of } \\
\text { another participant. It can be words like "Hey", "Oh", "Ah", etc., or the } \\
\text { name of the other speaker. }\end{array}$ \\
\hline Social Obligation & $\begin{array}{l}\text { This type of discourse relation is used when a participant complies with } \\
\text { certain social norms or obligations, such as apologies, acceptance or rejec- } \\
\text { tion of apologies, appreciation, greetings, farewell, etc. When a participant } \\
\text { is signaling their desire for ending a conversation, that message is consid- } \\
\text { ered farewell, and is thus labeled as Social Obligation. }\end{array}$ \\
\hline Other & $\begin{array}{l}\text { Occasionally, a participant might send an empty message, and in that } \\
\text { case, the relation of the empty message to its immediate previous message } \\
\text { should be annotated as Other. Other is also used when a given message } \\
\text { is nonsensical in relation to any previous message, or when the relation } \\
\text { between two messages are not formalized in any of the categories above. }\end{array}$ \\
\hline
\end{tabular}

Figure 4: Dialogue and Non-Dialogue Labels

of "scrambling" is even higher between differentparticipant message pairs, where one participant is responding to a message of another participant.

The inter-annotator agreement statistics for the four rounds of annotation are presented in Table 1. Column 4 shows the agreement on connections only, which is computed as the percentage of messages that are linked to the same antecedent for both annotators. Column 5 shows the agreement on relations, which is computed as the proportion of message pairs that are annotated with the same relation, out of the total number of connections that both annotators agree on. So this calculation factors out connections that the two annotators have disagreements on. Column 6 shows the Cohen's Kappa on relation agreement. The results show the agreement on connections stays relatively stable between rounds, indicating this aspect of the annotation is rather intuitive, and does not benefit from additional rounds of training. In contrast, there is significant improvement in the agreement on relations as guidelines are refined and the distinction between the relations are clarified. The final column shows the agreement on both connections and labels. The agreements statistics are lower, indicating a cumulative effect, but overall, it shows that reliable annotation can be achieved.

The inter-annotator agreement (IAA) statistics on connections are calculated with equation 3

$$
P=\frac{N_{a}}{N_{t}}
$$

where $N_{a}$ is the total number of same connections, and $N_{t}$ is the total number of connections. The inter-annotator agreement for connections with label is calculated similarly: $N_{a}$ is the total number of same connections with the same label.

The Cohen's Kappa score for labels on the same connections is calculated as follows:

$$
K=\frac{P_{o}-P_{e}}{1-P_{e}}
$$

where $P_{o}$ is the sum of probabilities of choosing the same label, and $P_{e}$ is the probability of choosing the same label by chance,

$$
P_{e}=\sum P_{i}^{a} \times P_{i}^{b}
$$

where $P_{i}^{a}$ and $P_{i}^{b}$ are the probabilities of annotator A and annotator B choosing label $i$, respectively. $P_{e}$ is the sum of the products of $P_{i}^{a}$ and $P_{i}^{b}$ for all labels.

\subsection{Examples of Inter-annotator Disagreement}

There are two main types of disagreement between the annotators: disagreement on connections and disagreement on relations. Disagreement on connections happens when, given a message, the annotators disagree on which previous message is its antecedent. Disagreement on relations occurs when the annotators disagree on the relation between a given pair of messages. 


\begin{tabular}{l|l|l|l|l} 
Distance & $\mathbf{1}$ & $\mathbf{2}$ & $\mathbf{3}$ & $\mathbf{4}$ and greater \\
\hline Dialogue Links & $73.30 \%$ & $17.08 \%$ & $5.72 \%$ & $3.88 \%$ \\
\hline $\begin{array}{l}\text { Non-dialogue } \\
\text { Links }\end{array}$ & $84.22 \%$ & $11.18 \%$ & $3.22 \%$ & $1.36 \%$ \\
\hline $\begin{array}{l}\text { Dialogue and } \\
\begin{array}{l}\text { Non-dialogue } \\
\text { Combined }\end{array}\end{array}$ & $77.97 \%$ & $14.56 \%$ & $4.65 \%$ & $2.80 \%$ \\
\hline
\end{tabular}

Figure 5: Distance Distributions

\begin{tabular}{l|l|l|l|l|l|l} 
& $\begin{array}{l}\text { Number of } \\
\text { Files }\end{array}$ & $\begin{array}{l}\text { Number of } \\
\text { Messages }\end{array}$ & $\begin{array}{l}\text { Agreement } \\
\text { on connec- } \\
\text { tions }\end{array}$ & $\begin{array}{l}\text { Agreement } \\
\text { on relations }\end{array}$ & $\begin{array}{l}\text { Kappa on } \\
\text { relations }\end{array}$ & $\begin{array}{l}\text { Agreement } \\
\text { on both }\end{array}$ \\
\hline Round 1 & 10 & 898 & 0.886 & 0.697 & 0.649 & 0.618 \\
Round 2 & 14 & 873 & 0.886 & 0.722 & 0.680 & 0.640 \\
Round 3 & 10 & 893 & 0.848 & 0.838 & 0.826 & 0.710 \\
Round 4 & 10 & 890 & 0.867 & 0.881 & 0.875 & 0.764 \\
\hline
\end{tabular}

Table 1: Inter-Annotator agreement statistics

\begin{tabular}{|l|l|l|l|}
\hline Message ID & Timestamp & Participant ID & Content \\
\hline m0007 & $2010-08-24$ 19:22:45 UTC & 153902 & $\begin{array}{l}\text { Charming is the audience's subjective } \\
\text { interpretation }\end{array}$ \\
\hline m0008 & $2010-08-24$ 19:22:49 UTC & 153901 & $\begin{array}{l}\text { so you can choose to be condescend- } \\
\text { ing? }\end{array}$ \\
\hline m0009 & $2010-08-2419: 23: 02$ UTC & 153902 & Yes \\
\hline m0010 & $2010-08-2419: 23: 06$ UTC & 153901 & but you cannot choose to be charming \\
\hline m0011 & $2010-08-2419: 23: 14$ UTC & 153902 & You can attempt to be charming \\
\hline
\end{tabular}

Figure 6:

Disagreement on connections Although determining which message is connected to which previous message is intuitive for the most part, disagreement does happen when a message has more than one possible and meaningful connection. For instance, message m0010 in Figure 6 can be a response to message m0009 or an extension of message m0008. This is one of the cases on which the two annotators disagree.

Disagreement on Relations Certain words or phrases are generally ambiguous and prone to causing confusion and disagreement on labeling. For example, the word "yeah" or the phrase "I know" can either signal acknowledgment or express agreement. Disagreement on labeling often occurs when such words or phrases can be interpreted either way in a given context. Message m0053 in Figure 7 can be either acknowledgment or agreement of the assertion in their previous message, and either interpretation makes sense in this context.

\section{Related work}

There has been relatively little work on annotating the discourse and dialogue structure of SMS conversations. The work that is most similar to ours is that of (Perret, 2015), where they annotated the discourse structure of multi-party dialogues using a corpus collected from an on-line version of the The Settlers of Catan game. They argue that multi-party dialogues need to be modeled with a graph structure and adopted an annotation scheme in the SDRT framework (Asher and Lascarides, 2003). In our annotation, since we are dealing with SMS dialogues that involve two participants, we did not find a graph structure to be necessary. We opted for a simpler (non-projective) dependency structure that is easier to model algorithmically. In fact, (Perret, 2015) developed an automatic discourse parser based on the Maximum Spanning Tree, a tree-based dependency parsing algorithm (McDonald, 2006) instead of a graphbased algorithm. We also make a distinction be- 


\begin{tabular}{|l|l|l|l|}
\hline Message ID & Timestamp & Participant ID & Content \\
\hline m0051 & $2015-02-27$ 13:45:13 UTC & 152252 & It's so stupid Sofie \\
\hline m0052 & $2015-02-27$ 13:45:36 UTC & 152252 & $\begin{array}{l}\text { I just feel like the general public should } \\
\text { take an art class }\end{array}$ \\
\hline m0053 & $2015-02-27$ 13:45:36 UTC & 152212 & i know \\
\hline
\end{tabular}

Figure 7: Disagreement on relations

tween same-participant and different-participant relations, and argue SMS message conversations need to be modeled with an annotation framework based on both discourse coherence and dialogue structures.

\section{Conclusion and Future Work}

In this paper we presented a framework for annotating the discourse and dialogue structure of SMS message conversations. The annotation specifications integrate elements of coherence-based discourse relations and dialogue structure in conversational speech. We conducted annotation experiments that show reliable annotation. Future work includes additional annotation based on this annotation framework and producing sufficient data that can be used to train a statistical parsing model.

\section{Acknowledgment}

We gratefully acknowledge a Faculty/Student Collaborative Research Grant from the Office of the Dean of College of Arts and Science Dean's at Brandeis University that has supported the first and third author of this paper. All errors and shortcomings are that of the authors of course.

\section{References}

Nicholas Asher and Alex Lascarides. 2003. Logics of Conversation. Cambridge University Press.

Lynn Carlson, Daniel Marcu, and Mary Ellen Okurowski. 2001. Building a discourse-tagged corpus in the framework of rhetorical structure theory. In Proceedings of the Second SIGdial Workshop on Discourse and Dialogue - Volume 16, SIGDIAL '01, pages 1-10, Stroudsburg, PA, USA. Association for Computational Linguistics.

Mark G Core and James Allen. 1997. Coding dialogs with the damsl annotation scheme. In AAAI fall symposium on communicative action in humans and machines, pages 28-35. Boston, MA.

The PDTB Research Group. 2008. The PDTB 2.0. annotation manual. Technical Report IRCS-08-01, page 28 .
Ryan McDonald. 2006. Discriminative Training and Spanning Tree Algorithms for Dependency Parsing. Ph.D. thesis, University of Pennsylvania.

Stergos Afantenos Eric Kow Nicholas Asher Jérémy Perret. 2015. Discourse parsing for multi-party chat dialogues. In Proceedings of the 2015 Conference on Empirical Methods in Natural Language Processing, Lisbon, Portugal.

Rashmi Prasad, Nikhil Dinesh, Alan Lee, Eleni Miltsakaki, Livio Robaldo, Aravind Joshi, and Bonnie Webber. 2008. The Penn Discourse Treebank 2.0. In Proceedings of the 6th International Conference on Language Resources and Evaluation (LREC 2008), Marrakech, Morocco.

Andreas Stolcke, Noah Coccaro, Rebecca Bates, Paul Taylor, Carol Van Ess-Dykema, Klaus Ries, Elizabeth Shriberg, Daniel Jurafsky, Rachel Martin, and Marie Meteer. 2000. Dialogue act modeling for automatic tagging and recognition of conversational speech. Computational linguistics, 26(3):339-373. 